Proceedings of the Edinburgh Mathematical Society (2004) 47, 669-678 (C)

DOI:10.1017/S0013091502000275 Printed in the United Kingdom

\title{
*-REPRESENTATION TYPE OF *-DOUBLES OF FINITE-DIMENSIONAL ALGEBRAS
}

\author{
VOLODYMYR MAZORCHUK ${ }^{1}$ AND LYUDMILA TUROWSKA ${ }^{2}$ \\ ${ }^{1}$ Matematiska Institutionen, Uppsala Universitet, Box 480, \\ SE 751 06, Uppsala, Sweden (mazor@math.uu.se) \\ ${ }^{2}$ Chalmers Tekniska Högskola, Matematik, SE 412 96, \\ Göteborg, Sweden (turowska@math.chalmers.se)
}

(Received 23 February 2002)

Dedicated to Professor Yuriu Samoŭlenko on his 60th birthday

\begin{abstract}
We determine when the $*$-double of a finite-dimensional complex algebra is $*$-finite, $*$-tame and $*$-wild.

Keywords: *-algebra; representation; tame algebra; wild algebra
\end{abstract}

2000 Mathematics subject classification: Primary 46K10; 16G60

\section{Introduction}

Let $\mathcal{A}_{n}^{(1)}$ and $\mathcal{A}_{n}^{(2)}$ be free algebras over $\mathbb{C}$ with $n$ generators, $x_{1}^{(\varepsilon)}, \ldots, x_{n}^{(\varepsilon)}, \varepsilon=1,2$, respectively. Denote by $\sigma: \mathcal{A}_{n}^{(1)} \rightarrow \mathcal{A}_{n}^{(2)}$ the unique anti-homomorphism (that is, a map satisfying $\sigma(\lambda a+\mu b)=\bar{\lambda} \sigma(a)+\bar{\mu} \sigma(b), \sigma(a b)=\sigma(b) \sigma(a)$ for all $\left.\lambda, \mu \in \mathbb{C}, a, b \in \mathcal{A}_{n}^{(1)}\right)$ such that $\sigma\left(x_{j}^{(1)}\right)=x_{j}^{(2)}$ for all $j=1, \ldots, n$.

Let $\mathcal{A}$ be a finite-dimensional algebra, such that $\mathcal{A} \simeq \mathcal{A}_{n}^{(1)} / I$ for some ideal $I \in \mathcal{A}_{n}^{(1)}$. Then the set $\sigma(I)$ is an ideal in $\mathcal{A}_{n}^{(2)}$ and we can consider the algebra $\mathcal{A}^{*}=\mathcal{A}_{n}^{(2)} / \sigma(I)$. It is easy to see that $\mathcal{A}^{*}$ does not depend on the presentation of $\mathcal{A}$.

Consider now the quotient algebra $\mathcal{A}(*)$ of the free product $\mathcal{A}_{n}^{(1,2)}$ of $\mathcal{A}_{n}^{(1)}$ and $\mathcal{A}_{n}^{(2)}$ (i.e. the free algebra with $2 n$ generators $\left.x_{1}^{(1)}, \ldots, x_{n}^{(1)}, x_{1}^{(2)}, \ldots, x_{n}^{(2)}\right)$ by the ideal $J$ generated by $I$ and $\sigma(I)$. The algebra $\mathcal{A}(*)$ is identified with the free product of $\mathcal{A}$ and $\mathcal{A}^{*}$ in a natural way. The algebra $\mathcal{A}_{n}^{(1,2)}$ possesses a natural $*$-structure, defined by $\left(x_{j}^{(1)}\right)^{*}=x_{j}^{(2)}$, $j=1, \ldots, n$, and one sees that $J$ is a $*$-ideal with respect to this structure. Hence $\mathcal{A}(*)$ inherits the $*$-structure and we call $\mathcal{A}(*)$ with this $*$-structure the $*$-double of $\mathcal{A}$. The algebra $\mathcal{A}(*)$ together with the natural inclusion algebra morphism $\mathfrak{i}: \mathcal{A} \rightarrow \mathcal{A}(*)$ defined via $\mathfrak{i}\left(x_{1}^{(1)}\right)=x_{1}^{(1)}$ is characterized by the following universal property: if $\mathcal{B}$ is any *-algebra and $\varphi: \mathcal{A} \rightarrow \mathcal{B}$ is an algebra morphism, then there exists a unique morphism 
$\hat{\varphi}: \mathcal{A}(*) \rightarrow \mathcal{B}$ of $*$-algebras, which makes the following diagram commutative:

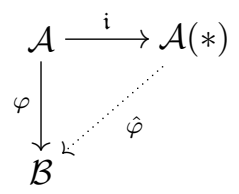

Representations of $*$-doubles of some particular algebras appeared and were investigated in $[\mathbf{1}, \mathbf{6}, \mathbf{9}]$ (see also $[\mathbf{8}]$ and the references therein). The results obtained showed that except for a few cases the representations have a very complicated structure (the corresponding $*$-algebras are $*$-wild) indicating that it could be a common feature. The aim of this note is to give a complete classification for the $*$-representation type of the algebras $\mathcal{A}(*)$ into $*$-finite, $*$-tame and $*$-wild types, answering a question posed by Samoilenko (personal communication). We show that, as for finite-dimensional non-involutive algebras (see $[\mathbf{3}]$ ), $*$-doubles of finite-dimensional algebras can be subdivided into $*$-tame (including $*$-finite) and $*$-wild $*$-algebras. Moreover, the subdivision is determined by the dimension of the original algebra. Using this result we derive some corollaries on representation type of $*$-doubles of not necessarily finite-dimensional associative algebra, in particular, we prove that the $*$-double of a finite-dimensional Lie algebra (i.e. of the corresponding universal enveloping algebra) is $*$-wild.

The paper is organized as follows: in $\S 2$ we recall basic definitions on $*$-wild and $*$-tame algebras and fix the notation. In $\S 3$ we formulate our main results, which are proved in $\S 5$ after some auxiliary lemmas collected in $\S 4$.

All algebras in this paper are over $\mathbb{C}$ and have a unit. All tensor products are taken over $\mathbb{C}$.

\section{2. *-Wild and $*$-tame algebras}

In this section we list some notation and definitions of $*$-wild and $*$-tame algebras following [6]. Within this section we assume the following convention: all $*$-algebras are unital with the unit $e$ and representations of $*$-algebras are unital $*$-homomorphisms into $B(H)$, the $*$-algebra of linear bounded operators on a separable Hilbert space, $H$. We denote by $\operatorname{Rep}(\mathcal{A})$ the category of all representations of a $*$-algebra, $\mathcal{A}$. Given a $*$-algebra, $\mathcal{A}$, of operators on $H$, we denote its commutator by $\mathcal{A}^{\prime}$, i.e. $\mathcal{A}^{\prime}=\{C \in B(H) \mid[C, A]=$ 0 , for each $A \in \mathcal{A}$ \}.

Let $\mathcal{A}$ be a $*$-algebra. We call a pair $(\tilde{\mathcal{A}} ; \phi: \mathcal{A} \rightarrow \tilde{\mathcal{A}})$, where $\tilde{\mathcal{A}}$ is a $*$-algebra and $\phi$ is a unital $*$-homomorphism, an enveloping $*$-algebra of the algebra $\mathcal{A}$ if, for any $*$ representation $\pi: \mathcal{A} \rightarrow B(H)$ of $\mathcal{A}$, there exists a unique $*$-representation $\tilde{\pi}: \tilde{\mathcal{A}} \rightarrow B(H)$ such that the diagram

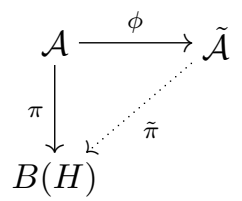


is commutative, and any operator $X: H_{1} \rightarrow H_{2}$ which intertwines representations $\pi_{1}$ : $\mathcal{A} \rightarrow B\left(H_{1}\right)$ and $\pi_{2}: \mathcal{A} \rightarrow B\left(H_{2}\right)$ of $\mathcal{A}$ is an intertwining operator for the representations $\tilde{\pi}_{1}$ and $\tilde{\pi}_{2}$ of the algebra $\tilde{\mathcal{A}}$.

Let $M_{n}(\mathcal{A})\left(=M_{n}(\mathbb{C}) \otimes \mathcal{A}\right)$ be the matrix algebra over $\mathcal{A}$. If $\mathcal{A}$ is a $C^{*}$-algebra, then $M_{n}(\mathcal{A})$ carries the natural structure of a $C^{*}$-algebra too. Let $\pi: \mathcal{A} \rightarrow B(H)$ be a representation of $\mathcal{A}$. It induces the representation $\pi_{n}: M_{n}(\mathcal{A}) \rightarrow B(H \oplus \cdots \oplus H)$ of the algebra $M_{n}(\mathcal{A})$. The representation $\pi_{n}$ determines the representation $\tilde{\pi}_{n}$ of an enveloping algebra $\left(\tilde{M}_{n}(\mathcal{A}), \phi\right)$ of $M_{n}(\mathcal{A})$ on the same Hilbert space. If $\psi$ is a unital $*$-homomorphism of a $*$-algebra $\mathcal{B}$ to the algebra $\tilde{M}_{n}(\mathcal{A})$, then $\tilde{\pi}_{n} \circ \psi$ defines a representation of $\mathcal{B}$. So we can define a functor, $F_{\psi}: \operatorname{Rep}(\mathcal{A}) \rightarrow \operatorname{Rep}(\mathcal{B})$, in the following natural way:

(i) $F_{\psi}(\pi)=\tilde{\pi}_{n} \circ \psi$, for every $\pi \in \operatorname{Rep}(\mathcal{A})$;

(ii) $F_{\psi}(c)=\operatorname{diag}(c, \ldots, c)$ for a morphism, $c: \pi_{1} \rightarrow \pi_{2}$, of representations $\pi_{1}, \pi_{2}$ of $\mathcal{A}$.

We say that a $*$-algebra $\mathcal{B}$ majorizes a $*$-algebra $\mathcal{A}(\mathcal{B} \succ \mathcal{A})$ if there exist $n \in$ $\mathbb{N}$, an enveloping algebra, $\tilde{M}_{n}(\mathcal{A})$, of the algebra $M_{n}(\mathcal{A})$, and a $*$-homomorphism $\psi: \mathcal{B} \rightarrow \tilde{M}_{n}(\mathcal{A})$ such that the functor $F_{\psi}: \operatorname{Rep}(\mathcal{A}) \rightarrow \operatorname{Rep}(\mathcal{B})$ is full. Note that the functor $F_{\psi}$ is automatically faithful. Clearly, in this case two representations $\pi_{1}, \pi_{2}$ of $\mathcal{A}$ are unitarily equivalent if and only if the representations $F_{\psi}\left(\pi_{1}\right), F_{\psi}\left(\pi_{2}\right)$ of $\mathcal{B}$ are unitarily equivalent, a representation $\pi$ of $\mathcal{A}$ is irreducible if and only if the representation $F_{\psi}(\pi)$ is irreducible. Thus the problem of unitary classification of the representations of the $*$-algebra $\mathcal{B}$ contains, as a subproblem, the problem of unitary classification of the representations of the $*$-algebra $\mathcal{A}$. Note that, in order to verify that the functor $F_{\psi}$ is full, it is sufficient to show that for each representation $\pi \in \operatorname{Rep} \mathcal{A}$ on $H$ and $C \in B(H)$ the inclusion $C \in F_{\psi}(\pi)(\mathcal{B})^{\prime}$ implies $C=\operatorname{diag}(c, \ldots, c)$, where $c \in \pi(\mathcal{A})^{\prime}$. We also note that the majorization of $*$-algebras is a quasi-order relation: if $\mathcal{C} \succ \mathcal{B}$ and $\mathcal{B} \succ \mathcal{A}$, then $\mathcal{C} \succ \mathcal{A}$ (see $[\mathbf{8}]$ for details).

It was proved in $[\mathbf{5}, \mathbf{6}]$ that $\mathfrak{S}_{2}=\mathbb{C}\left\langle a_{1}, a_{2} \mid a_{1}=a_{1}^{*}, a_{2}=a_{2}^{*}\right\rangle$ majorizes any finitely generated $*$-algebra and therefore the $C^{*}$-algebra $C^{*}\left(\mathcal{F}_{2}\right)$ of the free group $\mathcal{F}_{2}$ with two generators. Besides, $C^{*}\left(\mathcal{F}_{2}\right) \succ \mathfrak{S}_{2}$, motivating the following definition.

Definition 2.1. A *-algebra $\mathcal{A}$ is called $*$-wild if $\mathcal{A} \succ C^{*}\left(\mathcal{F}_{2}\right)$.

By the arguments given above, a $*$-algebra $\mathcal{A}$ is $*$-wild if and only if $\mathcal{A} \succ \mathfrak{S}_{2}$. For other results and examples on $*$-wild algebras we refer the reader to $[6,8]$.

Definition 2.2. A $*$-algebra is called $*$-finite if it has only finitely many irreducible representations up to unitary equivalence, and $*$-tame if it is of type $I$ and not $*$-finite (see $[\mathbf{2}])$.

Note that every finitely generated $*$-algebra whose irreducible representations are finite-dimensional is of type $I[\mathbf{2}]$.

\section{Main results}

Our main result is the following. 
Theorem 3.1. Let $\mathcal{A}$ be a finite-dimensional algebra over $\mathbb{C}$. Then

(1) the $*$-algebra $\mathcal{A}(*)$ is $*$-finite if and only if $\operatorname{dim}(\mathcal{A})=1$ if and only if $\mathcal{A} \simeq \mathbb{C}$;

(2) the $*$-algebra $\mathcal{A}(*)$ is $*$-tame if and only if $\operatorname{dim}(\mathcal{A})=2$ if and only if $\mathcal{A} \simeq \mathbb{C} \oplus \mathbb{C}$ or $\mathcal{A} \simeq \mathbb{C}[X] /\left(X^{2}\right)$

(3) the $*$-algebra $\mathcal{A}(*)$ is $*$-wild if and only if $\operatorname{dim}(\mathcal{A})>2$.

From Theorem 3.1 one easily derives the following two corollaries.

Corollary 3.2. If $\mathcal{A}$ is an associative algebra which has a finite-dimensional irreducible representation of dimension $n>1$ or a finite-dimensional indecomposable representation of dimension $n>2$, then $\mathcal{A}(*)$ is $*$-wild.

Corollary 3.3. The $*$-double of the universal enveloping algebra of a finite-dimensional Lie algebra is $*$-wild.

The proof of all these results will require several auxiliary lemmas, which we collect in the next section. The results themselves will be proved in $\S 5$.

\section{Auxiliary lemmas}

Lemma 4.1. The $*$-algebra $M_{n}(\mathbb{C})(*)$ is $*$-wild for $n>1$.

Proof. We consider first the case $n=2$ and denote by $e_{s t}, s, t \in\{1,2\}$, the canonical generators of $M_{2}(\mathbb{C})$. We define the $*$-homomorphism $\psi_{2}: M_{2}(\mathbb{C})(*) \rightarrow M_{2}\left(\mathfrak{S}_{2}\right)$ by

$$
\begin{array}{ll}
\psi_{2}\left(e_{11}\right)=\left(\begin{array}{cc}
e & -a_{1}-\mathrm{i} a_{2} \\
0 & 0
\end{array}\right), & \psi_{2}\left(e_{12}\right)=\left(\begin{array}{cc}
0 & e \\
0 & 0
\end{array}\right), \\
\psi_{2}\left(e_{21}\right)=\left(\begin{array}{cc}
a_{1}+\mathrm{i} a_{2} & -\left(a_{1}+\mathrm{i} a_{2}\right)^{2} \\
e & -a_{1}-\mathrm{i} a_{2}
\end{array}\right), & \psi_{2}\left(e_{22}\right)=\left(\begin{array}{cc}
0 & a_{1}+\mathrm{i} a_{2} \\
0 & e
\end{array}\right) .
\end{array}
$$

If we have a $2 \times 2$ self-adjoint operator matrix commuting with $\pi_{2}\left(\psi_{2}\left(e_{12}\right)\right)$, $\pi_{2}\left(\psi_{2}\left(e_{12}\right)^{*}\right)$, where $\pi \in \operatorname{Rep} \mathfrak{S}_{2}$, then it must be block diagonal, with the same operators on the diagonal, say $c$. Commuting it further with $\pi_{2}\left(\psi_{2}\left(e_{11}\right)\right)$ and the corresponding adjoint we get $c\left(\pi\left(a_{1}\right) \pm \mathrm{i} \pi\left(a_{2}\right)\right)=\left(\pi\left(a_{1}\right) \pm \mathrm{i} \pi\left(a_{2}\right)\right) c$, and therefore $\left[c, \pi\left(a_{i}\right)\right]=0$ proving that the corresponding functor $F_{\psi}: \operatorname{Rep} \mathfrak{S}_{2} \rightarrow \operatorname{Rep} M_{2}(\mathbb{C})(*)$ is full and the $*$-algebra $M_{2}(\mathbb{C})(*)$ is $*$-wild.

If $n>2$, we define $\psi_{n}: M_{n}(\mathbb{C})(*) \rightarrow M_{n}\left(\mathfrak{S}_{2}\right)$ via

$$
\psi_{n}\left(e_{t s}\right)= \begin{cases}\psi_{2}\left(e_{t s}\right) \oplus 0_{n-2}, & \text { if } t, s \in\{1,2\}, \\ \left(\psi_{2}\left(e_{21}\right) \oplus 0_{n-2}\right) E_{1 s}^{(n)}, & \text { if } t=2, s>2, \\ E_{t 1}^{(n)}\left(\psi_{2}\left(e_{11}\right) \oplus 0_{n-2}\right), & \text { if } t>2, s=1, \\ E_{t s}^{(n)}, & \text { otherwise, }\end{cases}
$$


where $E_{t s}^{(n)}=e_{t s} \otimes e \in M_{n}(\mathbb{C}) \otimes \mathfrak{S}_{2}$. To see that $\psi_{n}$ is a $*$-homomorphism we have to show that $\psi_{n}\left(e_{k l}\right) \psi_{n}\left(e_{t s}\right)=\delta_{l t} \psi_{n}\left(e_{k s}\right)$. This clearly holds if both pairs $(k, l)$ and $(t, s)$ differ from $(m, 1)$ and $(2, m), m \leqslant n$. The relations which have to be checked are

$$
\begin{array}{ll}
\psi_{n}\left(e_{m 1}\right) \psi_{n}\left(e_{t s}\right)=\delta_{1 t} \psi_{n}\left(e_{m s}\right), & \psi_{n}\left(e_{t s}\right) \psi_{n}\left(e_{m 1}\right)=\delta_{s m} \psi_{n}\left(e_{t 1}\right), \\
\psi_{n}\left(e_{2 m}\right) \psi_{n}\left(e_{t s}\right)=\delta_{m t} \psi_{n}\left(e_{2 s}\right), & \psi_{n}\left(e_{t s}\right) \psi_{n}\left(e_{2 m}\right)=\delta_{s 2} \psi_{n}\left(e_{t m}\right) .
\end{array}
$$

We restrict ourselves to verifying the first one leaving the rest to the reader.

All entries of the matrix $\psi_{n}\left(e_{m 1}\right)$ are zero except for the first two in the $n$th row if $m \neq 2$ and the first two in the first and the second rows if $m=2$. Therefore, $\psi_{n}\left(e_{m 1}\right) \psi_{n}\left(e_{t s}\right)$ might be non-zero only if $t=1$ or $t=2$. Clearly, if $m, t, s \in\{1,2\}$, the relation is satisfied and hence we can assume that one of the indexes is not equal to 1 or 2 . A direct calculation shows that $\psi_{n}\left(e_{11}\right) \psi_{n}\left(e_{1 s}\right)=\psi_{n}\left(e_{1 s}\right)$ for any $s>1 . \psi_{n}\left(e_{21}\right) \psi_{n}\left(e_{1 s}\right)=\psi_{n}\left(e_{2 s}\right)$ holds by the definition of $\psi_{n}\left(e_{2 s}\right)$ for $s>2$. If $m>2$, we have that

$$
\psi_{n}\left(e_{m 1}\right)=E_{m 1}^{(n)} \psi_{n}\left(e_{11}\right) \text { and } \psi_{n}\left(e_{m 1}\right) \psi_{n}\left(e_{1 s}\right)=E_{m 1}^{(n)} \psi_{n}\left(e_{11}\right) \psi_{n}\left(e_{1 s}\right)=E_{m 1}^{(n)} \psi_{n}\left(e_{1 s}\right) \text {, }
$$

which is equal to $E_{m 1}^{(n)} E_{1 s}^{(n)}=E_{m s}^{(n)}=\psi_{n}\left(e_{m s}\right)$ if $s>1$ and to $\psi_{n}\left(e_{m 1}\right)$ if $s=1$ by the definition. Thus $\psi_{n}\left(e_{m 1}\right) \psi_{n}\left(e_{1 s}\right)=\psi_{n}\left(e_{m s}\right)$. If $s>2$ and $m=1,2$, then

$$
\psi_{n}\left(e_{m 1}\right) \psi_{n}\left(e_{2 s}\right)=\psi_{n}\left(e_{m 1}\right) \psi_{n}\left(e_{21}\right) E_{1 s}^{(n)}=0 .
$$

For $m>2$ we have that $\psi_{n}\left(e_{m 1}\right) \psi_{n}\left(e_{2 s}\right)=E_{m 1}^{(n)} \psi_{n}\left(e_{11}\right) \psi_{n}\left(e_{2 s}\right)=0$ by the previous relation and the relation $\psi_{n}\left(e_{11}\right) \psi_{n}\left(e_{2 s}\right)=0$ for $s=1,2$. Therefore, $\psi_{n}\left(e_{m 1}\right) \psi_{n}\left(e_{2 s}\right)=0$ for every $m, s$ and $\psi_{n}\left(e_{m 1}\right) \psi_{n}\left(e_{t s}\right)=\delta_{1 t} \psi_{n}\left(e_{m s}\right)$ for each $m, t$ and $s$.

To see that the functor $F_{\psi_{n}}$ is full, consider a representation, $\pi$, of $\mathfrak{S}_{2}$ on $H$ and a self-adjoint bounded operator, $C \in B(H)$, which commutes with each $\pi_{n}\left(\psi_{n}\left(e_{t s}\right)\right)$ and their adjoint. As $C$ commutes with $\pi_{n}\left(\psi_{n}\left(e_{t s}\right)\right)=\pi_{n}\left(E_{t s}^{(n)}\right), t, s>2$, writing $C$ as an $n \times n$ operator matrix, $C=\left[c_{t s}\right]_{t, s=1}^{n}$, we obtain that $c_{t s}=0$ if $t \neq s$ and if one of $t$ and $s$ is greater than 2 , and $c_{t t}=c_{s s}$ for $t, s>2$. Since $C$ commutes with $\pi_{n}\left(\psi_{n}\left(e_{t s}\right)\right)$ for $t, s \in\{1,2\}$, we get $c_{11}=c_{22}, c_{12}=c_{21}=0$ and $\left[c_{11}, a_{i}\right]=0$ so that the matrix $C$ is diagonal. That $C=\operatorname{diag}\left(c_{11}, \ldots, c_{11}\right)$ follows from the relation

$$
\left[C, \pi_{n}\left(\psi_{n}\left(e_{13}\right)\right)\right]=\left[C, \pi_{n}\left(E_{13}^{(n)}\right)\right]=0,
$$

giving us $c_{11}=c_{33}$.

Lemma 4.2 (see $\S 3.1 .3$ in [8]). The $*$-algebra $\mathfrak{Q}_{n, \perp}(*)$, where

$$
\left.\mathfrak{Q}_{n, \perp}=\mathbb{C}\left\langle q_{1}, \ldots, q_{n}\right| q_{i}^{2}=q_{i}, i=1, \ldots, n, q_{i} q_{j}=q_{j} q_{i}=0, \text { for } j \neq i\right\rangle,
$$

is $*$-wild for $n \geqslant 2$.

Proof. We define the homomorphism $\psi: \mathfrak{Q}_{n, \perp}(*) \rightarrow M_{3}\left(\mathfrak{S}_{2}\right)$ as follows:

$$
\psi\left(q_{1}\right)=\left(\begin{array}{ccc}
e & e & a_{1}+\mathrm{i} a_{2} \\
0 & 0 & 0 \\
0 & 0 & 0
\end{array}\right), \quad \psi\left(q_{2}\right)=\left(\begin{array}{ccc}
0 & -e & -e \\
0 & e & e \\
0 & 0 & 0
\end{array}\right), \quad \psi\left(q_{i}\right)=0, \quad i>2 .
$$


We leave it to the reader to check that $\psi$ is a homomorphism and the generated functor $F_{\psi}: \operatorname{Rep} \mathfrak{S}_{2} \rightarrow \operatorname{Rep} \mathfrak{Q}_{n, \perp}(*)$ is full.

Lemma 4.3. The $*$-algebra $\mathcal{B}(*)$, where $\mathcal{B}=\mathbb{C}[x] /\left(x^{2}-\alpha e\right), \alpha \in \mathbb{C}$, is $*$-tame. Every irreducible representation of $\mathcal{B}(*)$ is either one or two dimensional.

Proof. Set $a=\operatorname{Re} x=\left(x+x^{*}\right) / 2$ and $b=\operatorname{Im} x=\left(x-x^{*}\right) / 2$ i. Clearly, $a$ and $b$ are selfadjoint. Moreover, $x^{2}=\alpha e$ and $\left(x^{*}\right)^{2}=\bar{\alpha} e$ imply $a^{2}-b^{2}=(\operatorname{Re} \alpha) e$ and $a b+b a=(\operatorname{Im} \alpha) e$. By $[\mathbf{8}, \S 1.2 .3]$ or $[\mathbf{7}]$, every irreducible representation of these relations is either one or two dimensional and therefore the corresponding $*$-algebra is $*$-tame.

In the lemmas that follow we will need invertibility of $a_{1}$ and $a_{2}$ and positivity of their images under each bounded representation in order to be able to prove that the functors which we define are full. This means that we will not be able to work with the algebra $\mathfrak{S}_{2}$ any more, so we shall substitute for it the algebra $\mathfrak{C}$ (defined below), obtained from $\mathfrak{S}_{2}$ by imposing formal conditions of invertibility and positivity for $a_{1}$ and $a_{2}$. This algebra happens to be $*$-wild, as we will show in the next lemma. Let us consider, for some fixed $0<m<n$, the seminorm $\|a\|=\|a\|_{m, n}=\sup \pi(a)$ on the $*$-algebra $\mathfrak{S}_{2}$, where the supremum is taken over all representations $\pi$ of $\mathfrak{S}_{2}$ such that $m I \leqslant \pi\left(a_{i}\right) \leqslant n I, i=1,2$, $I$ being the identity operator. Denote by

$$
\mathfrak{C}=\mathfrak{C}_{m, n}=C^{*}\left(a_{1}, a_{2}: m \leqslant a_{i}=a_{i}^{*} \leqslant n, i=1,2\right)
$$

the $C^{*}$ algebra which is obtained by the completion of $\mathfrak{S}_{2} /(a:\|a\|=0)$ with respect to $\|\cdot\|$. Clearly, the elements $a_{1}$ and $a_{2}$ become invertible in $\mathfrak{C}$ and positive in every bounded representation. In the lemma that follows we prove that $\mathfrak{C}$ is actually $*$-wild.

Lemma 4.4. The algebra $\mathfrak{C}$ is $*$-wild.

Proof. Define the homomorphism $\psi: \mathfrak{C} \rightarrow M_{4}\left(C^{*}\left(\mathcal{F}_{2}\right)\right)$ as follows:

$$
\psi\left(a_{1}\right)=\operatorname{diag}\left(\lambda_{1} e, \lambda_{2} e, \lambda_{3} e, \lambda_{4} e\right),
$$

where $m \leqslant \lambda_{i} \leqslant n, i=1,2,3,4$, are distinct complex numbers, and

$$
\psi\left(a_{2}\right)=\frac{1}{\beta}\left(\begin{array}{cccc}
\alpha e & u^{*} & e & e \\
u & \alpha e & v^{*} & e \\
e & v & \alpha e & e \\
e & e & e & \alpha e
\end{array}\right),
$$

where $u, v$ are the generators of the $C^{*}$-algebra $C^{*}\left(\mathcal{F}_{2}\right)$ and $\alpha, \beta \in \mathbb{R}$ are chosen so that $m E_{4} \leqslant \psi\left(a_{2}\right) \leqslant n E_{4}$, where $E_{4}=\operatorname{diag}(e, e, e, e)$, in $M_{4}\left(C^{*}\left(\mathcal{F}_{2}\right)\right)$. That this can always be done follows by the following arguments. The element $\psi\left(a_{2}\right)$ with $\alpha=0$ and $\beta=1$ is, in fact, a self-adjoint element of the $C^{*}$-algebra $M_{4}\left(C^{*}\left(\mathcal{F}_{2}\right)\right)$. It has a norm, say $K$, and $-K e \leqslant \psi\left(a_{2}\right) \leqslant K e$ in general. So, adding some $\alpha E_{4}$ to $\psi\left(a_{2}\right)$, we can ensure $\psi\left(a_{2}\right)>0$ and then, dividing by some positive constant, we get it in the required interval. It is now routine to check that the functor $F_{\psi}$ is full. 
Lemma 4.5. The *-algebra $\mathcal{A}_{R_{n}}(*)$, where $\mathcal{A}_{R_{n}}=\mathbb{C}\left\langle x \mid R_{n}(x)=0\right\rangle$ and $R_{n}$ is a complex polynomial in one variable of degree $n$, is $*$-wild if and only if $n \geqslant 3$.

Proof. By Lemma $4.3, \mathcal{A}_{R_{2}}(*)$ is $*$-tame. Clearly, it is enough to show that $\mathcal{A}_{R_{3}}(*)$ is $*$-wild (see Lemma 4.10 below). Assume first that $R_{3}$ has distinct roots: $R_{3}(x)=$ $\left(x-\alpha_{1}\right)\left(x-\alpha_{2}\right)\left(x-\alpha_{3}\right), \alpha_{1}, \alpha_{2}, \alpha_{3} \in \mathbb{C}, \alpha_{i} \neq \alpha_{j}$ for $i \neq j$. Defining the homomorphism $\psi: \mathcal{A}_{R_{3}}(*) \rightarrow \mathfrak{Q}_{2, \perp}(*)$ by setting

$$
\psi(x)=\alpha q_{1}+\alpha_{2} q_{2}+\alpha_{3}\left(e-q_{1}-q_{2}\right),
$$

one easily checks that the corresponding functor $F_{\psi}: \operatorname{Rep} \mathfrak{Q}_{2, \perp}(*) \rightarrow \operatorname{Rep} \mathcal{A}_{R_{3}}(*)$ is full and therefore $\mathcal{A}_{R_{3}}(*)$ majorizes the $*$-algebra $\mathfrak{Q}_{2, \perp}(*)$. Since majorization is a quasiorder, we obtain from Lemma 4.2 that $\mathcal{A}_{R_{3}}(*)$ is $*$-wild.

If $R_{3}(x)=\left(x-\alpha_{1}\right)^{2}\left(x-\alpha_{2}\right), \alpha_{1}, \alpha_{2} \in \mathbb{C}$ are equal or distinct, then the homomorphism $\psi: \mathcal{A}_{R_{3}}(*) \rightarrow M_{3}(\mathfrak{C})$ defined by

$$
\psi(x)=\left(\begin{array}{ccc}
\alpha_{1} e & a_{1} & e \\
0 & \alpha_{1} e & a_{2} \\
0 & 0 & \alpha_{2} e
\end{array}\right)
$$

generates a full functor $F_{\psi}: \operatorname{Rep} \mathfrak{C} \rightarrow \operatorname{Rep} \mathcal{A}_{R_{3}}(*)$. In fact, let $\pi$ be a representation of $\mathfrak{C}$. To prove that $F_{\psi}$ is full it is enough to show that any operator $C=C^{*}=\left[c_{i j}\right]_{i, j=1}^{3}$, which intertwines the representation $\pi_{3} \circ \psi$ of $\mathcal{A}_{R_{3}}(*)$, is $\operatorname{diag}(c, c, c)$, where $c$ intertwines the representation $\pi$ of $\mathfrak{C}$. Taking into account that $\pi\left(a_{i}\right), i=1,2$, are invertible one gets that if $\left[C, \pi_{3}(\psi(x))\right]=0$, then $C=\operatorname{diag}\left(c_{11}, c_{22}, c_{11}\right)$ and $c_{11} \pi\left(a_{1}\right)=\pi\left(a_{1}\right) c_{22}$, $c_{22} \pi\left(a_{2}\right)=\pi\left(a_{2}\right) c_{11}$. Since $c_{11}$ and $c_{22}$ are necessarily self-adjoint, we obtain from this that $c_{11} \pi\left(a_{1}\right)^{2}=\pi\left(a_{1}\right)^{2} c_{11}$ and, therefore, by the positivity of the operator $\pi\left(a_{1}\right)$, $c_{11} \pi\left(a_{1}\right)=\pi\left(a_{1}\right) c_{11}$. Thus we have $\pi\left(a_{1}\right) c_{22}=c_{11} \pi\left(a_{1}\right)=\pi\left(a_{1}\right) c_{11}$ and, using that $\pi\left(a_{1}\right)$ is invertible, it follows that $c_{11}=c_{22}$, giving $\mathcal{A}_{R_{3}}(*) \succ \mathfrak{C}$. By Lemma 4.4, we have that $\mathcal{A}_{R_{3}}(*)$ is $*$-wild.

Remark 4.6. That $\mathcal{A}_{R_{n}}(*)$ is $*$-wild when $R_{n}$ has three or more distinct roots was proved in $[\mathbf{1}, \mathbf{8}]$.

Lemma 4.7. The $*$-algebra $\mathcal{B}(*)$, where $\mathcal{B}=\mathbb{C}[x, y] /\left(x^{2}, y^{2}, x y\right)$, is $*$-wild.

Proof. Define the homomorphism $\psi: \mathcal{B}(*) \rightarrow M_{2}(\mathfrak{C})$ in the following way:

$$
\psi(x)=\left(\begin{array}{cc}
0 & a_{1} \\
0 & 0
\end{array}\right), \quad \psi(y)=\left(\begin{array}{cc}
0 & a_{2} \\
0 & 0
\end{array}\right) .
$$

Using arguments similar to those used in Lemma 4.5 one obtains that the corresponding functor is full and therefore the $*$-algebra $\mathcal{B}(*)$ is $*$-wild.

Lemma 4.8. The $*$-algebra $\mathcal{B}(*)$, where $\mathcal{B}=\mathbb{C}\left\langle f, x \mid f^{2}=f, x f=(1-f) x=x\right\rangle$, is *-wild. 
Proof. The homomorphism $\psi: \mathcal{B}(*) \rightarrow M_{2}(\mathfrak{C})$, defined by

$$
\psi(f)=\left(\begin{array}{cc}
e & a_{1} \\
0 & 0
\end{array}\right), \quad \psi(x)=\left(\begin{array}{cc}
-a_{1} a_{2} & -a_{1} a_{2} a_{1} \\
a_{2} & a_{2} a_{1}
\end{array}\right),
$$

generates a full functor, $F_{\psi}: \operatorname{Rep} \mathfrak{C} \rightarrow \operatorname{Rep} \mathcal{B}(*)$.

Lemma 4.9. The $*$-algebra $\mathcal{B}(*)$, where $\mathcal{B}=\mathbb{C} \oplus \mathbb{C}[x] /\left(x^{2}\right)$, is $*$-wild.

Proof. $\mathcal{B}(*)$ is generated by the idempotent $f=(1,0)$, the element $x$ and their adjoints, subject to the relations $f^{2}=f, x^{2}=0, f x=x f=0$. We define $\psi: \mathcal{B}(*) \rightarrow$ $M_{4}(\mathfrak{C})$ by setting

$$
\psi(f)=\left(\begin{array}{cccc}
e & 0 & \lambda e & a_{1} \\
0 & e & 0 & \mu e \\
0 & 0 & 0 & 0 \\
0 & 0 & 0 & 0
\end{array}\right), \quad \psi(x)=\left(\begin{array}{cccc}
0 & 0 & 0 & -\lambda a_{2} \\
0 & 0 & 0 & 0 \\
0 & 0 & 0 & a_{2} \\
0 & 0 & 0 & 0
\end{array}\right) .
$$

We leave it to the reader to check that the functor $F_{\psi}$ is full.

We finish our preparation with the following obvious observation.

Lemma 4.10. Let $\mathcal{A}$ be a finite-dimensional algebra and $I$ an ideal in $\mathcal{A}$. If $\mathcal{A} / I(*)$ is $*$-wild, then $\mathcal{A}(*)$ is $*$-wild as well.

\section{Proof of the main results}

Let $\mathcal{B}=\mathcal{A} / R$, where $R=\operatorname{rad}(\mathcal{A})$ is the radical of $\mathcal{A}$. We first recall the well-know fact that in $\mathcal{A}$ one can always lift idempotents from quotient algebras modulo any ideal contained in $R$ (see, for example, $[4, \S 3.2]$ ). The algebra $\mathcal{B}$ is semi-simple, say

$$
\mathcal{B} \simeq \bigoplus_{i} M_{n_{i}}(\mathbb{C})
$$

By Lemma 4.10 , if $\mathcal{B}(*)$ is $*$-wild, so is the $*$-algebra $\mathcal{A}(*)$. If some of the $n_{i}$ in the above decomposition is greater than 1 , then from Lemmas 4.1 and 4.10 it follows that $\mathcal{B}(*)$ is $*$-wild. According to Lemma 4.2 , the algebra $\mathcal{B}(*)$ is also $*$-wild if all $n_{i}=1$ and the number of the components is greater than two. Otherwise, $\mathcal{B} \simeq \mathbb{C}$ or $\mathcal{B} \simeq \mathbb{C} \oplus \mathbb{C}$ (the corresponding $*$-doubles are tame), and we have to study these cases separately.

Assume first that $\mathcal{B} \simeq \mathbb{C}$ and consider the quotient $R / R^{2}$. If $\operatorname{dim}\left(R / R^{2}\right)>1$, then $R$ contains a subspace, say $J$, such that $J \supset R^{2}$ and the codimension of $J / R^{2}$ in $R / R^{2}$ is 2 . Since the only primitive idempotent of $\mathcal{A}$ is the identity, it follows that $J$ is an ideal of $\mathcal{A}$ and $\mathcal{A} / J \simeq \mathbb{C}[x, y] /\left(x^{2}, y^{2}, x y\right)$. From Lemmas 4.7 and 4.10 we get that $\mathcal{A}(*)$ is $*$-wild in this case. If $\operatorname{dim}\left(R / R^{2}\right)=1$, then $R$ is generated by one element, say $x$, as an algebra, and hence $\mathcal{A}$ is a quotient of the polynomial algebra $\mathbb{C}[x]$. Since $R$ is nilpotent, we have that $x^{n}=0$ for some $n>1$ and thus $\mathcal{A} \simeq \mathbb{C}[x] /\left(x^{n}\right)$. If $n=2$, i.e. $\mathcal{A}$ is two dimensional, then, by Lemma $4.3, \mathcal{A}(*)$ is $*$-tame. If $n>2$, 
then, by Lemmas 4.5 and $4.10, \mathcal{A}(*)$ is $*$-wild. If $\operatorname{dim}\left(R / R^{2}\right)=0$, we have $R=0$, $\mathcal{A}=\mathbb{C}$ and therefore $\mathcal{A}(*)$ is $*$-finite. Note that this is the only case when $\mathcal{A}(*)$ is finite dimensional.

Finally, assume that $\mathcal{B} \simeq \mathbb{C} \oplus \mathbb{C}$. In this case $\mathcal{A}$ has two orthogonal idempotents, say $a$ and $b, a+b=e$. If $R=0$, then $\mathcal{A}=\mathbb{C} \oplus \mathbb{C}(\mathcal{A}$ is two dimensional $)$ and the $*$-double $\mathcal{A}(*)$ is $*$-tame by Lemma 4.3. Assume now that there exists $x \in R \backslash R^{2}$ such that $x a=x$ and $b x=x($ or $a x=x$ and $x b=x)$. Let $\mathcal{A}^{\prime}$ denote the quotient of $\mathcal{A}$ by the ideal $J$, generated by $R^{2}, a R a$ and $b R b$. Then we have either $(x+J)(a+J)=x+J$ and $(b+J)(x+J)=x+J$ or $(x+J)(b+J)=x+J$ and $(a+J)(x+J)=x+J$. Moreover, any subspace of $\operatorname{rad}\left(\mathcal{A}^{\prime}\right)$ is an ideal of $\mathcal{A}^{\prime}$. Let $V \subset \operatorname{rad}\left(\mathcal{A}^{\prime}\right)$ be a subspace of codimension 1, which does not contain $x+J$. Then $\mathcal{A}^{\prime} / V \simeq \mathbb{C}\left\langle f, x \mid f^{2}=f, x f=(1-f) x=x\right\rangle$. This means that we can use Lemmas 4.8 and 4.10 and conclude that $\mathcal{A}^{\prime}(*)$ and hence $\mathcal{A}(*)$ are $*$-wild.

If the element $x$ with the properties above does not exist, we have that $a R b=b R a=0$, and hence, if $R$ is non-zero, we obtain that either $a R a /(a R a)^{2} \neq 0$ or $b R b /(b R b)^{2} \neq 0$, with both $a R a$ and $b R b$ being ideals in $\mathcal{A}$. Without loss of generality we can assume that $a R a /(a R a)^{2} \neq 0$. Then we find $V \subset a R a$ such that $V \supset(a R a)^{2}$ and the codimension of $V /(a R a)^{2}$ in $a R a /(a R a)^{2}$ is 1 . For the ideal $J=V+R^{2}+b R b$ we obtain that $\mathcal{A} / J=\mathbb{C}[x] /\left(x^{2}\right) \oplus \mathbb{C}$, whose $*$-double is $*$-wild by Lemma 4.9. This completes the proof of Theorem 3.1 .

Corollary 3.2 follows using the following arguments. The only associative algebra of dimension one is the field $\mathbb{C}$ itself, and for this one all indecomposable representations are irreducible and have dimension one. Any associative algebra of dimension two is commutative and thus isomorphic to $\mathbb{C}[x] /\left(x^{2}+a x+b\right)$ for some $a, b \in \mathbb{C}$. If the roots of $x^{2}+a x+b$ are different, the algebra is isomorphic to $\mathbb{C} \oplus \mathbb{C}$, which is semi-simple with one-dimensional irreducible representations, hence all indecomposable representations are again irreducible and have dimension 1 . Finally, if the roots of $x^{2}+a x+b$ coincide, we get an algebra, isomorphic to $\mathbb{C}[x] /\left(x^{2}\right)$, which has only one irreducible representation (up to isomorphism) of dimension one and one indecomposable non-irreducible representation of dimension 2 by the Jordan Theorem. Hence, under the assumptions of Corollary 3.2, we must have $\operatorname{dim}(\mathcal{A})>2$ and thus $\mathcal{A}(*)$ is $*$-wild by Theorem 3.1.

Corollary 3.3 is proved as follows. If $L$ is a finite-dimensional Lie algebra, then $L / \operatorname{rad}(L)$ is either semi-simple or zero. We have, therefore, the following two possibilities: (a) $L=\operatorname{rad}(L)$ and $L /[L, L]$ is abelian and non-zero, or (b) $L$ has a quotient, which is a non-zero simple Lie algebra. In the first case the universal enveloping algebra $U(L /[L, L])$ is a polynomial ring and hence the $*$-double of $U(L /[L, L])$ is $*$-wild (by, say, Lemmas 4.10 and 4.5). Therefore, $U(L)(*)$ is $*$-wild by Lemma 4.10. In the second case the algebra $U(L)$ has an irreducible representation of dimension greater than 1 and, by Corollary $3.2, U(L)(*)$ is *-wild.

Acknowledgements. Research was partially supported by the Royal Swedish Academy of Sciences. V.M.'s research was also partially supported by the Swedish Research Council. We thank Professor Samollenko for informing us about this problem and for helpful discussions. We also thank the referee for a number of helpful suggestions. 


\section{References}

1. Yu. N. Bespalov and Yu. S. SamoǏlenko,

Algebraic operators and pairs of selfadjoint operators connected with a polynomial relation, Funkt. Analysis Prilozhen. 25(4) (1991), 72-74 (in Russian; transl. Funct. Analysis Applic. 25 (1991/1992), 289-291).

2. J. Dixmier, $C^{*}$-algebras (North-Holland, Amsterdam, 1982).

3. Ju. A. Drozd, Tame and wild matrix problems, Representation theory, II, in Proc. 2nd Int. Conf. Carleton Univ., Ottawa, ON, 1979, pp. 242-258, Lecture Notes in Mathematics, vol. 832 (Springer, 1980).

4. Y. Drozd And V. KirichenKo, Finite-dimensional algebras (translated from the 1980 Russian original and with an appendix by Vlastimil Dlab) (Springer, 1994).

5. S. A. Kruglyak and Yu. S. Samol̆Lenko, Unitary equivalence of sets of selfadjoint operators, Funkt. Analysis Prilozhen. 14 (1980), 60-62 (in Russian).

6. S. A. Kruglyak and Yu. S. SamoĬlenko, On complexity of description of representation of *-algebras generated by idempotents, Proc. Am. Math. Soc. 128 (2000), 1655-1664.

7. V. OstrovskiI and Yu. SAmoĬLEnko, Representations of quadratic *-algebras by bounded and unbounded operators, Rep. Math. Phys. 35 (1995), 283-301.

8. V. Ostrovskil and YU. SAmol̆LenKo, Introduction to the representation theory of finitely presented $*$-algebras, 1 , Representations by bounded operators, Rev. Math. Math. Phys. 11 (1999), 1-261.

9. Yu. Samol̆LenKo and L. Turowska, On bounded and unbounded idempotents whose sum is a multiple of the identity, Meth. Funct. Analysis Topol. 8(1) (2002), 79-100. 